\title{
A limitation of the Bruggeman formalism for homogenization
}

\author{
Tom G. Mackay ${ }^{1}$ \\ School of Mathematics, University of Edinburgh, Edinburgh EH9 3JZ, UK \\ Akhlesh Lakhtakia ${ }^{2}$ \\ CATMAS - Computational $\&$ Theoretical Materials Sciences Group \\ Department of Engineering Science and Mechanics \\ Pennsylvania State University, University Park, PA 16802-6812, USA
}

\begin{abstract}
The Bruggeman formalism provides an estimate of the effective permittivity of a particulate composite medium comprising two component mediums. The Bruggeman estimate is required to lie within the Wiener bounds and the Hashin-Shtrikman bounds. Considering the homogenization of weakly dissipative component mediums characterized by relative permittivities with real parts of opposite signs, we show that the Bruggeman estimate may not be not physically reasonable when the component mediums are weakly dissipative; furthermore, both the Wiener bounds and the HashinShtrikman bounds exhibit strong resonances.
\end{abstract}

Keywords: homogenization; negative permittivity; Bruggeman formalism; Maxwell Garnett formalism; Hashin-Shtrikman bounds; Wiener bounds

\section{Introduction}

Metamaterials in the form of particulate composite mediums are currently of considerable scientific and technological interest [1]. Provided that wavelengths are sufficiently long compared with the length scales of inhomogeneities, such a metamaterial may be envisaged as a homogenized composite medium (HCM), arising from two homogeneous component mediums $[2,3]$. HCMs with especially interesting properties may be conceptualized if the real parts of the relative permittivities (and/or relative permeabilities) of the two component mediums have opposite signs [4]. This possibility arises for metal-in-insulator dielectric composites $[5,6]$ and has recently become feasible with the fabrication of dielectric-magnetic materials displaying a negative index of refraction in the microwave frequency range $[7,8]$.

\footnotetext{
${ }^{1}$ Corresponding Author. Fax: + 44131650 6553; e-mail: T.Mackay@ed.ac.uk.

${ }^{2}$ Fax:+1 814865 99974; e-mail: akhlesh@psu.edu
} 
Over many years, several theoretical formalisms have been developed in order to estimate the effective constitutive parameters of particulate composite mediums [2]. In particular, the Maxwell Garnett and the Bruggeman homogenization formalisms have been widely used [9]. Generally, the Maxwell Garnett formalism is seen to hold only for dilute composite mediums [10]. ${ }^{3}$ More widely applicable is the Bruggeman formalism that was initially founded on the intuition that the total polarization field is zero throughout the HCM [16]. A rigorous basis for the Bruggeman formalism is also available, within the framework of the strongpermittivity-fluctuation theory (SPFT) [17, 18].

Estimates of HCM constitutive parameters generated by homogenization formalisms may be required to lie within certain bounds. In particular, the Wiener bounds [19, 20] and the Hashin-Shtrikman bounds [21] are often invoked. The Hashin-Shtrikman bounds coincide with the constitutive parameter estimates of the Maxwell Garnett homogenization formalism [20]. The applicability of theoretical bounds on the HCM permittivity has recently been the focus of attention for composites specified by relative permittivities with positive-valued real parts $[22]$.

In this communication, we consider the application of the Bruggeman formalism, together with the Wiener and Hashin-Shtrikman bounds, to isotropic dielectric HCMs which arise from component mediums characterized by complex-valued relative permittivities whose real parts have opposite signs. This is scenario is typical of metal-in-insulator HCMs [20, 23], for example. By duality, our analysis extends to isotropic magnetic HCMs. It also extends to isotropic dielectric-magnetic HCMs, because the permeability and the permittivity are then independent of each other in the Bruggeman formalism [24] (as also in the Maxwell Garnett formalism [25]). Therefore, our findings are very relevant to the application of homogenization formalisms [4] to mediums displaying negative index of refraction [26], for example. Furthermore, the implications of our mathematical study extend beyond the Bruggeman formalism to the SPFT as well [3].

A note on notation: $A n \exp (-i \omega t)$ time-dependence is implicit in the following sections; and the real and imaginary parts of complex-valued quantities are denoted by $\operatorname{Re}(\cdot)$ and $\operatorname{Im}(\bullet)$, respectively.

\footnotetext{
${ }^{3}$ The restriction on the applicability of the Maxwell Garnett formalism to dilute composite mediums generally emerges from comparison with experimental data [9]. As the particulate volume fraction increases, the distribution of particles begins to lose the randomness which is inherent to the theory of the Maxwell Garnett formalism [11]. However, the restriction could be bypassed if the distribution of particles in a composite medium continues to lack order even under densification, which thought underlies the random unit cell approach developed by Smith and colleagues $[12,13,14]$. An anonymous reviewer has suggested that self-assembly techniques [15] can yield randomness even at large particulate volume fractions, and could therefore extend the applicability of the Maxwell Garnett formalism.
} 


\section{Theory}

\subsection{Bruggeman equation}

Consider the homogenization of two isotropic dielectric component mediums labelled $a$ and $b$. Let their relative permittivities be denoted by $\epsilon_{a}$ and $\epsilon_{b}$, respectively. For later convenience, we define

$$
\delta=\left\{\begin{array}{clr}
\frac{\epsilon_{a}}{\epsilon_{b}} & \text { if } & \epsilon_{a}, \epsilon_{b} \in \mathbb{R}, \\
\frac{\operatorname{Re}\left(\epsilon_{a}\right)}{\operatorname{Re}\left(\epsilon_{b}\right)} & \text { if } & \epsilon_{a}, \epsilon_{b} \in \mathbb{C} .
\end{array}\right.
$$

The Bruggeman estimate of the HCM relative permittivity, namely $\epsilon_{\mathrm{HCM}}^{\mathrm{Br}}$, is provided implicitly via the relation [9]

$$
\epsilon_{\mathrm{HCM}}^{\mathrm{Br}}=\frac{f_{a} \epsilon_{a}\left(\epsilon_{b}+2 \epsilon_{\mathrm{HCM}}^{\mathrm{Br}}\right)+f_{b} \epsilon_{b}\left(\epsilon_{a}+2 \epsilon_{\mathrm{HCM}}^{\mathrm{Br}}\right)}{f_{a}\left(\epsilon_{b}+2 \epsilon_{\mathrm{HCM}}^{\mathrm{Br}}\right)+f_{b}\left(\epsilon_{a}+2 \epsilon_{\mathrm{HCM}}^{\mathrm{Br}}\right)}
$$

wherein $f_{a}$ and $f_{b}$ are the respective volume fractions of component mediums $a$ and $b$, and the particles of both component mediums are assumed to be spherical. The Bruggeman equation (2) emerges naturally within the SPFT framework [3]. A rearrangement of (2) gives the quadratic equation

$$
2\left(\epsilon_{\mathrm{HCM}}^{\mathrm{Br}}\right)^{2}+\epsilon_{\mathrm{HCM}}^{\mathrm{Br}}\left[\epsilon_{a}\left(f_{b}-2 f_{a}\right)+\epsilon_{b}\left(f_{a}-2 f_{b}\right)\right]-\epsilon_{a} \epsilon_{b}=0 .
$$

Only those $\epsilon_{\mathrm{HCM}}^{\mathrm{Br}}$-solutions of (3) are valid under the principle of causality as encapsulated by the Kramers-Kronig relations [28] which conform to the restriction $\operatorname{Im}\left(\epsilon_{\mathrm{HCM}}^{\mathrm{Br}}\right) \geq 0$.

Let $\Delta$ be the discriminant of the quadratic equation (3); i.e.,

$$
\Delta=\left[\epsilon_{a}\left(f_{b}-2 f_{a}\right)+\epsilon_{b}\left(f_{a}-2 f_{b}\right)\right]^{2}+8 \epsilon_{a} \epsilon_{b} .
$$

Since $f_{b}=1-f_{a}$, we may express (4) as

$$
\Delta=9 f_{a}^{2}\left(\epsilon_{a}-\epsilon_{b}\right)^{2}-6 f_{a}\left(\epsilon_{a}^{2}-3 \epsilon_{a} \epsilon_{b}+2 \epsilon_{b}^{2}\right)+\left(\epsilon_{a}+2 \epsilon_{b}\right)^{2} .
$$

An insight into the applicability of the Bruggeman formalism may be gained by considering the $f_{a}$-roots of the equation $\Delta=0$; these are as follows:

$$
\left.f_{a}\right|_{\Delta=0}=\frac{\epsilon_{a}^{2}-3 \epsilon_{a} \epsilon_{b}+2 \epsilon_{b}^{2} \pm 2 \sqrt{2} \sqrt{-\epsilon_{a} \epsilon_{b}\left(\epsilon_{a}-\epsilon_{b}\right)^{2}}}{3\left(\epsilon_{a}-\epsilon_{b}\right)^{2}} .
$$

On restricting attention to nondissipative component mediums (i.e., $\epsilon_{a, b} \in \mathbb{R}$ ), it is clear that $\left.f_{a}\right|_{\Delta=0}$ are complex-valued if $\delta>0$. Consequently, $\Delta>0$ which implies that $\epsilon_{\mathrm{HCM}}^{\mathrm{Br}} \in \mathbb{R}$. On the other hand, $\left.f_{a}\right|_{\Delta=0}$ are real-valued if $\delta<0$. Thus, the Bruggeman estimate $\epsilon_{\mathrm{HCM}}^{\mathrm{Br}}$ for $\delta<0$ may be complex-valued with nonzero imaginary part, even though neither component medium is dissipative. 


\subsection{Bounds on the HCM relative permittivity}

Various bounds on the HCM relative permittivity have been developed. Two of the most widely used are the Wiener bounds [19, 20]

$$
\left.\begin{array}{l}
W_{\alpha}=\left(\frac{f_{a}}{\epsilon_{a}}+\frac{f_{b}}{\epsilon_{b}}\right)^{-1} \\
W_{\beta}=f_{a} \epsilon_{a}+f_{b} \epsilon_{b}
\end{array}\right\}
$$

and the Hashin-Shtrikman bounds [21]

$$
\left.\begin{array}{l}
H S_{\alpha}=\epsilon_{b}+\frac{3 f_{a} \epsilon_{b}\left(\epsilon_{a}-\epsilon_{b}\right)}{\epsilon_{a}+2 \epsilon_{b}-f_{a}\left(\epsilon_{a}-\epsilon_{b}\right)} \\
H S_{\beta}=\epsilon_{a}+\frac{3 f_{b} \epsilon_{a}\left(\epsilon_{b}-\epsilon_{a}\right)}{\epsilon_{b}+2 \epsilon_{a}-f_{b}\left(\epsilon_{b}-\epsilon_{a}\right)}
\end{array}\right\} .
$$

While both the Wiener bounds and the Hashin-Shtrikman bounds were originally derived for real-valued constitutive parameters, generalizations to complex-valued constitutive parameters have been established [23].

The Hashin-Shtrikman bound $\mathrm{HS}_{\alpha}$ is equivalent to the Maxwell Garnett estimate of the HCM relative permittivity $\epsilon_{\mathrm{HCM} / a}^{\mathrm{MG}}$ based on spherical particles of component medium $a$ embedded in the host component medium $b$. Similarly, $\mathrm{HS}_{\beta}$ is equivalent to the Maxwell Garnett estimate of the HCM relative permittivity $\epsilon_{\mathrm{HCM} / b}^{\mathrm{MG}}$ based on spherical particles of component medium $b$ embedded in the host component medium $a$. The estimate $\epsilon_{\mathrm{HCM} / a}^{\mathrm{MG}}$ is valid for $f_{a} \lesssim 0.3$, whereas the estimate $\epsilon_{\mathrm{HCM} / b}^{\mathrm{MG}}$ is valid for $f_{b} \lesssim 0.3$; but see the footnote in Section 1.

To gain insights into the asymptotic behaviour of the Wiener and Hashin-Shtrikman bounds, let us again restrict attention to the case of nondissipative component mediums (i.e., $\epsilon_{a, b} \in \mathbb{R}$ ). From $(7)$, we see that $W_{\beta}$ remains finite for all values of $\delta$, but $W_{\alpha}$ may become infinite for $\delta<0$ since

$$
\left|W_{\alpha}\right| \rightarrow \infty \quad \text { as } \quad \delta \rightarrow-\frac{f_{a}}{f_{b}} .
$$

In a similar vein, from (8) we find that

$$
\left|H S_{\alpha}\right| \rightarrow \infty \quad \text { as } \quad \delta \rightarrow \frac{f_{b}-3}{f_{b}}
$$

thus, for all values of $\delta<-2$ there exists a value of $f_{b} \in(0,1)$ at which $H S_{\alpha}$ is unbounded. Analogously,

$$
\left|H S_{\beta}\right| \rightarrow \infty \quad \text { as } \quad \delta \rightarrow \frac{f_{a}}{f_{a}-3}
$$

so we can always find a value of $f_{a} \in(0,1)$ at which $H S_{\beta}$ is unbounded, provided that $\delta \in\left(-\frac{1}{2}, 0\right)$. 


\section{Numerical results}

Let us now present, calculated values of the HCM relative permittivity $\epsilon_{\mathrm{HCM}}^{\mathrm{Br}}$, along with the corresponding values of the bounds $W_{\alpha, \beta}$ and $H S_{\alpha, \beta}$, for some representative examples. Both nondissipative and dissipative HCMs are considered for $\delta= \pm 3$.

\subsection{Nondissipative component mediums}

The effects of dissipation may be very clearly appreciated through first considering the idealized situation wherein the components mediums are nondissipative [27]. Furthermore, although the absence of dissipation is unphysical due to the dictates of causality [28], weak dissipation in a particular spectral regime is definitely possible and is then often ignored $[9$, Sec.2.5].

Thus, it is instructive to begin with the commonplace scenario wherein both $\epsilon_{a}>0$ and $\epsilon_{b}>0$. For example, let $\epsilon_{a}=6$ and $\epsilon_{b}=2$. In Figure $1, \epsilon_{\mathrm{HCM}}^{\mathrm{Br}}$ is plotted against $f_{a}$, along with the corresponding Wiener bounds $W_{\alpha, \beta}$ and Hashin-Shtrikman bounds $H S_{\alpha, \beta}$. The latter bounds are stricter than the former bounds in the sense that

$$
W_{\alpha}<H S_{\alpha}<\epsilon_{\mathrm{HCM}}^{\mathrm{Br}}<H S_{\beta}<W_{\beta}
$$

The close agreement between $\epsilon_{\mathrm{HCM}}^{\mathrm{Br}}$ and the lower Hashin-Shtrikman bound $H S_{\alpha}$ at low volume fractions $f_{a}$ is indicative of the fact that $H S_{\alpha} \equiv \epsilon_{\mathrm{HCM} / a}^{\mathrm{MG}}$. Similarly, $\epsilon_{\mathrm{HCM}}^{\mathrm{Br}}$ agrees closely with the upper Hashin-Shtrikman bound $H S_{\beta}$ at high values of $f_{a}$ since $H S_{\beta} \equiv \epsilon_{\mathrm{HCM} / b}^{\mathrm{MG}}$.

A markedly different situation develops if the real-valued $\epsilon_{a}$ and $\epsilon_{b}$ have opposite signs. For example, the values of $\epsilon_{\mathrm{HCM}}^{\mathrm{Br}}$ calculated for $\epsilon_{a}=-6$ and $\epsilon_{b}=2$ are graphed against $f_{a}$ in Figure 2, together with the corresponding Wiener and Hashin-Shtrikman bounds. The Bruggeman estimate $\epsilon_{\mathrm{HCM}}^{\mathrm{Br}}$ is complex-valued with nonzero imaginary part for $f_{a} \lesssim 0.82$. This estimate is not physically reasonable. The Bruggeman homogenization formalism unlike the SPFT which is its natural generalization - has no mechanism for taking coherent scattering losses into account. Furthermore, no account has been taken in the Bruggeman equation (2) for the finite size of the particles [11, 29, 30]. Therefore, the Bruggeman estimate of the HCM relative permittivity is required to be real-valued if the component mediums are nondissipative.

While $\epsilon_{\mathrm{HCM}}^{\mathrm{Br}}$ in Figure 2 is complex-valued, the Wiener bounds and the Hashin-Shtrikman bounds are both real-valued. In accordance with (9), we see that $\left|W_{\alpha}\right| \rightarrow \infty$ as $f_{a} \rightarrow \frac{3}{4}$. Similarly, $\left|H S_{\alpha}\right| \rightarrow \infty$ in the limit $f_{a} \rightarrow \frac{1}{4}$, as may be anticipated from (10). Furthermore, since $H S_{\alpha} \equiv \epsilon_{\mathrm{HCM} / a}^{\mathrm{MG}}$, the Maxwell Garnett formalism is clearly inappropriate here. We also observe that the inequalities (12) which hold for $\delta>0$, do not hold for $\delta<0$.

\subsection{Weakly dissipative component mediums}

Let us now investigate $\epsilon_{\mathrm{HCM}}^{\mathrm{Br}}$ and its associated bounds when the component mediums are dissipative; i.e., $\epsilon_{a, b} \in \mathbb{C}$. We begin with those cases for which $\delta>0$ : for example, we take 
$\epsilon_{a}=6+0.3 i$ and $\epsilon_{b}=2+0.2 i$. In Figure $3, \epsilon_{\mathrm{HCM}}^{\mathrm{Br}}$ is plotted against $f_{a}$, and the associated Wiener bounds $W_{\alpha, \beta}$ and the Hashin-Shtrikman bounds $H S_{\alpha, \beta}$ are also presented. The behaviour of the real parts of $\epsilon_{\mathrm{HCM}}^{\mathrm{Br}}, W_{\alpha, \beta}$ and $H S_{\alpha, \beta}$ closely resembles that displayed in the nondissipative example of Figure 1. In fact, the following generalization of (12) holds:

$$
\operatorname{Re}\left(W_{\alpha}\right)<\operatorname{Re}\left(H S_{\alpha}\right)<\operatorname{Re}\left(\epsilon_{\mathrm{HCM}}^{\mathrm{Br}}\right)<\operatorname{Re}\left(H S_{\beta}\right)<\operatorname{Re}\left(W_{\beta}\right) .
$$

However, this ordering (13) does not extend to the imaginary parts of $\epsilon_{\mathrm{HCM}}^{\mathrm{Br}}, W_{\alpha, \beta}$ and $H S_{\alpha, \beta}$.

Turning to the cases for $\delta<0$, we let $\epsilon_{a}=-6+0.3 i$ and $\epsilon_{b}=2+0.2 i$, for example. The corresponding Bruggeman estimate $\epsilon_{\mathrm{HCM}}^{\mathrm{Br}}$ is graphed as function of $f_{a}$, along with the Wiener bounds $W_{\alpha, \beta}$ and the Hashin-Shtrikman bounds $H S_{\alpha, \beta}$ in Figure 4 . Since $\operatorname{Im}\left(\epsilon_{a, b}\right) \neq 0$, the real parts of $W_{\alpha}$ and $H S_{\alpha}$ remain finite, unlike in the corresponding nondissipative scenario presented in Figure 2.

However, the real and imaginary parts of $W_{\alpha}$ and $H S_{\alpha}$ exhibit strong resonances in the vicinity of $f_{a}=\frac{3}{4}$ (for $W_{\alpha}$ ) and $f_{a}=\frac{1}{4}$ (for $H S_{\alpha}$ ). These resonances become considerably more pronounced if the degree of dissipation exhibited by the component mediums is reduced. For example, in Figure 5 the graphs corresponding to Figure 4 are reproduced for $\epsilon_{a}=$ $-6+0.003 i$ and $\epsilon_{b}=2+0.002 i$. We observe in particular that $\operatorname{Im}\left(\epsilon_{\mathrm{HCM}}^{\mathrm{Br}}\right)>1$ for $0.05 \lesssim$ $f_{a} \lesssim 0.8$. Thus, the Bruggeman estimate $\epsilon_{\mathrm{HCM}}^{\mathrm{Br}}$ vastly exceeds both the Wiener bounds $W_{\alpha, \beta}$ and the Hashin-Shtrikman bounds $H S_{\alpha, \beta}$ for a wide range of $f_{a}$. Since $\operatorname{Im}\left(\epsilon_{a, b}\right) \leq 0.003$, the estimates of $\operatorname{Im}\left(\epsilon_{\mathrm{HCM}}^{\mathrm{Br}}\right)$ are clearly unreasonable.

Furthermore, since the real and imaginary parts of $H S_{\alpha} \equiv \epsilon_{\mathrm{HCM} / a}^{\mathrm{MG}}$ exhibit sharp resonances at $f_{a}=\frac{1}{4}$, we may infer that the Maxwell Garnett formalism is inapplicable for $\delta<0$.

\subsection{Highly dissipative component mediums}

On comparing Figures 4 and 5, we conclude that the Bruggeman formalism, the Weiner bounds and the Hashin-Shtrikman bounds become increasing inappropriate as the degree of dissipation decreases towards zero. This means that all three could be applicable rather well when the dissipation is not weak.

Therefore, let us examine the scenario wherein the real and imaginary parts of the relative permittivities of the component medium are of the same order of magnitude; i.e., we take $\epsilon_{a}=-6+3 i$ and $\epsilon_{b}=2+2 i$. The corresponding plots of the Bruggeman estimate $\epsilon_{\mathrm{HCM}}^{\mathrm{Br}}$ together with the Wiener bounds $W_{\alpha, \beta}$ and the Hashin-Shtrikman bounds $H S_{\alpha, \beta}$ are presented in Figure 6. The real and imaginary parts of the Bruggeman estimate are physically plausible, and both lie within the Hashin-Shtrikman bounds. The Hashin-Shtrikman bounds themselves do not exhibit resonances, and the Weiner bounds do not exhibit strong resonances. Accordingly, we conclude that many previously published results are not erroneous, but caution is still advised. 


\section{Discussion}

The Bruggeman homogenization formalism is well-established in the context of isotropic dielectric HCMs, as well as more generally [2]. However, this formalism was shown in Section 3.2 to be inapplicable for HCMs which arise from two isotropic dielectric component mediums, characterized by relative permittivities $\epsilon_{a}$ and $\epsilon_{b}$, with

(i) $\operatorname{Re}\left(\epsilon_{a}\right)$ and $\operatorname{Re}\left(\epsilon_{b}\right)$ having opposite signs; and

(ii) $\left|\operatorname{Re}\left(\epsilon_{a, b}\right)\right| \gg\left|\operatorname{Im}\left(\epsilon_{a, b}\right)\right|$.

Since the Bruggeman formalism provides the comparison medium which underpins the SPFT, it may be inferred that the SPFT is likewise not applicable to the scenarios of (i) with (ii).

It is also demonstrated in Section 3.2 that both the Wiener bounds and the HashinShtrikman bounds can exhibit strong resonances when the component mediums are characterized by (i) with (ii). In the vicinity of resonances, these bounds clearly do not constitute tight bounds on the HCM relative permittivity. As a direct consequence, the Maxwell Garnett homogenization formalism, like the Bruggeman homogenization formalism, is inapplicable to the scenarios of (i) with (ii). This limitation also extends to the recently developed incremental [31] and differential [32] variants of the Maxwell Garnett formalism.

If the component mediums are sufficiently dissipative then the Bruggeman formalism and the Hashin-Shtrikman bounds (and therefore also the Maxwell Garnett formalism) provide physically plausible estimates, despite the real parts of the component medium relative permittivities having opposite signs - as shown in Section 3.3. The explicit delineation of the appropriate parameter range(s) for the Bruggeman formalism and the Hashin-Shtrikman bounds is a matter for future investigation.

Bounds can, of course, be violated by a formalism if the underlying conditions for the formalism are in conflict with those used for deriving the bounds. Sihvola [22] has catalogued the following conflicts:

(a) Bounds derived for nondissipative component mediums can be invalid for the real parts of either $\epsilon_{\mathrm{HCM} / a}^{\mathrm{MG}}$ or $\epsilon_{\mathrm{HCM} / b}^{\mathrm{MG}}$ for a composite medium containing dissipative component mediums.

(b) Percolation cannot be cannot be captured by the Maxwell Garnett formalism [5, 33]. Hence, the Hashin-Shtrikman bounds, being based on the Maxwell Garnett formalism, can be violated by the Bruggeman estimate $\epsilon_{\mathrm{HCM}}^{\mathrm{Br}}$ for a percolative composite medium.

(c) The derivations of bounds generally assume that the particles in a composite medium have simple shapes. If the particle shapes are complicated, the composite medium may display properties not characteristic of the either of the component mediums. For instance, magnetic properties can be displayed when the particles in a composite medium have complex shapes $[34,35]$, even though the component mediums are nonmagnetic. Clearly, the magnetic analogs of $W_{\alpha}, W_{\beta}, H S_{\alpha}$ and $H S_{\beta}$ are then inapplicable. 
(d) $W_{\alpha}, W_{\beta}, H S_{\alpha}$, and $H S_{\beta}$ as well as their magnetic analogs are also invalid prima facie when the component mediums exhibit magnetoelectric properties $[3,11,36]$.

(e) Bounds derived for electrically small particles become inapplicable with increasing frequency, due to the emergence of finite-size effects [29]. Even the concept of homogenization becomes questionable with increasing electrical size [2, p. xiii].

In contrast, the bounds and the homogenization formalisms studied in this paper share the same premises; yet, a conflict arises in certain situations because the bounds exhibit resonance while the homogenization estimates do not.

\section{Concluding remarks}

As several conventional approaches to homogenization are not appropriate to the HCMs arising from component mediums characterized by (i) with (ii), there is a requirement for new theoretical techniques to treat this case. This requirement is all the more pressing, given the growing scientific and technological importance of new types of metamaterials $[1,26]$.

Acknowledgement. We thank two anonymous reviewers for comments that led to the improvement of this paper.

\section{References}

[1] R.M. Walser, in: W.S. Weiglhofer, A. Lakhtakia (Eds.), Introduction to Complex Mediums for Optics and Electromagnetics, SPIE, Bellingham, WA, USA, 2003, pp.295-316.

[2] A. Lakhtakia (Ed.), Selected Papers on Linear Optical Composite Materials, SPIE, Bellingham, WA, USA, 1996.

[3] T.G. Mackay, in: W.S. Weiglhofer, A. Lakhtakia (Eds.), Introduction to Complex Mediums for Optics and Electromagnetics, SPIE, Bellingham, WA, USA, 2003, pp.317-345.

[4] A. Lakhtakia, Int. J. Infrared Millim. Waves 23 (2002) 813.

[5] J.A. Sherwin, A. Lakhtakia, B. Michel, Opt. Commun. 178 (2000) 267.

[6] T.G. Mackay, A. Lakhtakia, W.S. Weiglhofer, Opt. Commun. 197 (2001) 89.

[7] R.A. Shelby, D.R. Smith, S. Schultz, Science 292 (2001) 77.

[8] D.R. Smith, W.J. Padilla, D.C. Vier, S.C. Nemat-Nasser, S. Schultz, Phys. Rev. Lett. 84 (2000) 4184.

[9] L. Ward, The Optical Constants of Bulk Materials and Films, 2nd Edition, Adam Hilger, Bristol, UK, 1995; Chap. 8. 
[10] J.C. Maxwell Garnett, Phil. Trans. R. Soc. Lond. A 203 (1904) 385. (Reproduced in $[2])$.

[11] A. Lakhtakia, B. Shanker, Int. J. Appl. Electromag. Mater. 4 (1993) 65.

[12] G.B. Smith, J. Phys. D: Appl. Phys. 10 (1977) L39.

[13] G.A. Niklasson, C.G. Granqvist, O. Hunderi, Appl. Opt. 20 (1981) 26.

[14] G.B. Smith, in: W.S. Weiglhofer, A. Lakhtakia (Eds.), Introduction to Complex Mediums for Optics and Electromagnetics, SPIE, Bellingham, WA, USA, 2003, pp.421-446.

[15] S. Zhang, Materials Today 6(5) (2003), 20; May issue.

[16] D.A.G. Bruggeman, Ann. Phys. Lpz. 24 (1935) 636. (Reproduced in [2]).

[17] L. Tsang, J.A. Kong, Radio Sci. 16 (1981) 303. (Reproduced in [2]).

[18] T.G. Mackay, A. Lakhtakia, W.S. Weiglhofer, Phys. Rev. E 62 (2000) 6052; Erratum 63 (2001) 049901.

[19] O. Wiener, Abh. Math.-Phys. Kl. Sächs. 32 (1912) 507. (Reproduced in [2]).

[20] D.E. Aspnes, Am. J. Phys. 50 (1982) 704. (Reproduced in [2]).

[21] Z. Hashin, S. Shtrikman, J. Appl. Phys. 33 (1962) 3125.

[22] A.H. Sihvola, IEEE Trans. Geosci. Remote Sens. 40 (2002) 880.

[23] G.W. Milton, Appl. Phys. Lett. 37 (1980) 300.

[24] R.D. Kampia, A. Lakhtakia, J. Phys. D: Appl. Phys. 25 (1992) 1390.

[25] A. Lakhtakia, Int. J. Electron. 73 (1992) 1355.

[26] A. Lakhtakia, M.W. McCall, W.S. Weiglhofer, in: W.S. Weiglhofer, A. Lakhtakia (Eds.), Introduction to Complex Mediums for Optics and Electromagnetics, SPIE, Bellingham, WA, USA, 2003, pp.347-363.

[27] H.C. van de Hulst, Light Scattering by Small Particles, Dover, New York, NY, USA, 1981; Chap. 10.

[28] C.F. Bohren, D.R. Huffman, Absorption and Scattering of Light by Small Particles, Wiley, New York, NY, USA, 1983; Sec. 2.3.2.

[29] M.T. Prinkey, A. Lakhtakia, B. Shanker, Optik 96 (1994) 25.

[30] B. Shanker, J. Phys. D: Appl. Phys. 29 (1996) 281. 
[31] A. Lakhtakia, Microw. Opt. Technol. Lett. 17 (1998) 276.

[32] B. Michel, A. Lakhtakia, W.S. Weiglhofer, T.G. Mackay, Compos. Sci. Technol. 61 (2001) 13.

[33] A. Sihvola, S. Saastamoinen, K. Heiska, Remote Sens. Rev. 9 (1994) 39.

[34] A. Lakhtakia, Beltrami Fields in Chiral Media, World Scientific, Singapore, 1994; Chap. 3.

[35] J.B. Pendry, A.J. Holden, D.J. Robbins, W.J. Stewart, IEEE Trans. Microw. Theory Tech. 47 (1999) 2075.

[36] B. Michel, in: O.N. Singh, A. Lakhtakia (Eds.), Electromagnetic Fields in Unconventional Materials and Structures, Wiley, New York, NY, USA, 2000, pp.39-82. 


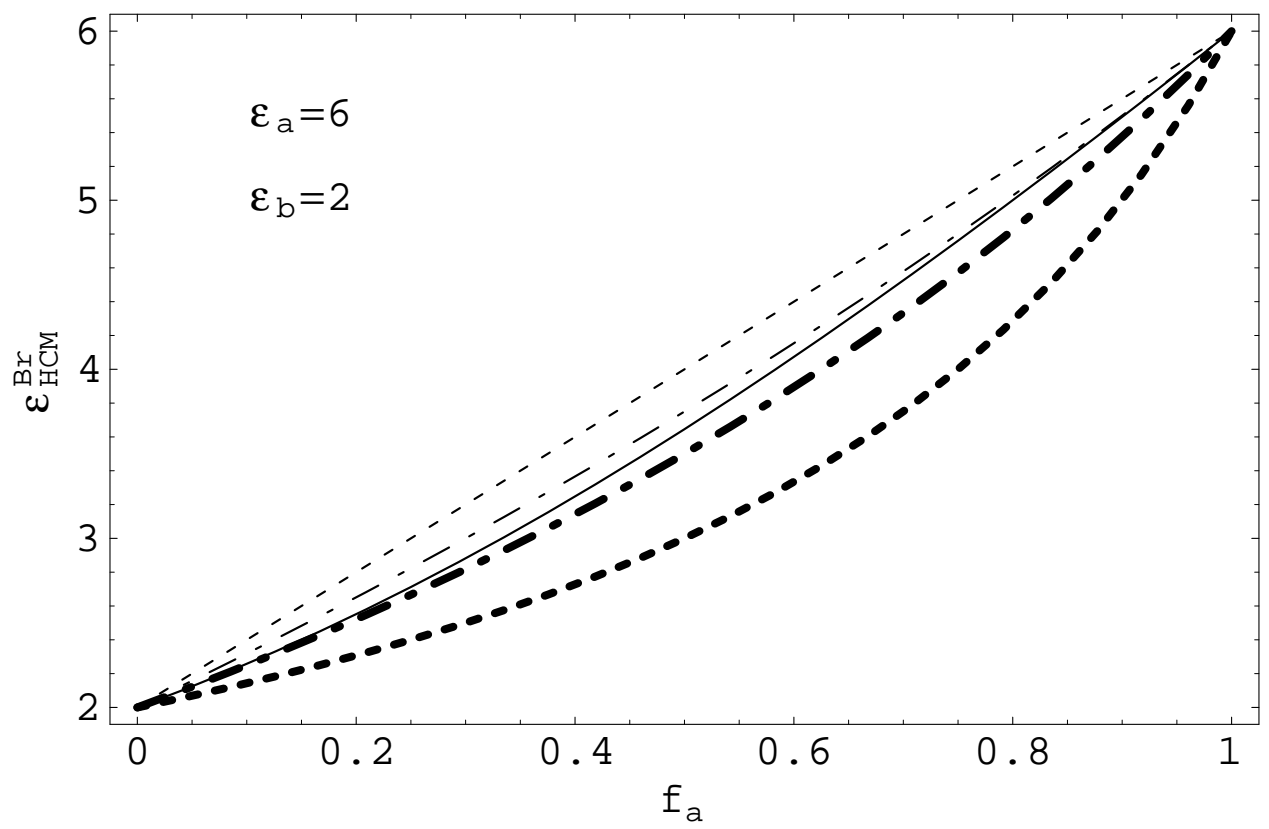

Figure 1: The Bruggeman estimate $\epsilon_{\mathrm{HCM}}^{\mathrm{Br}}$ (solid line) plotted against $f_{a}$ for $\epsilon_{a}=6$ and $\epsilon_{b}=2$. Also plotted are the Wiener bounds, $W_{\alpha}$ (thick dashed line) and $W_{\beta}$ (thin dashed line), and the Hashin-Shtrikman bounds, $H S_{\alpha}$ (thick broken dashed line) and $H S_{\beta}$ (thin broken dashed line). 

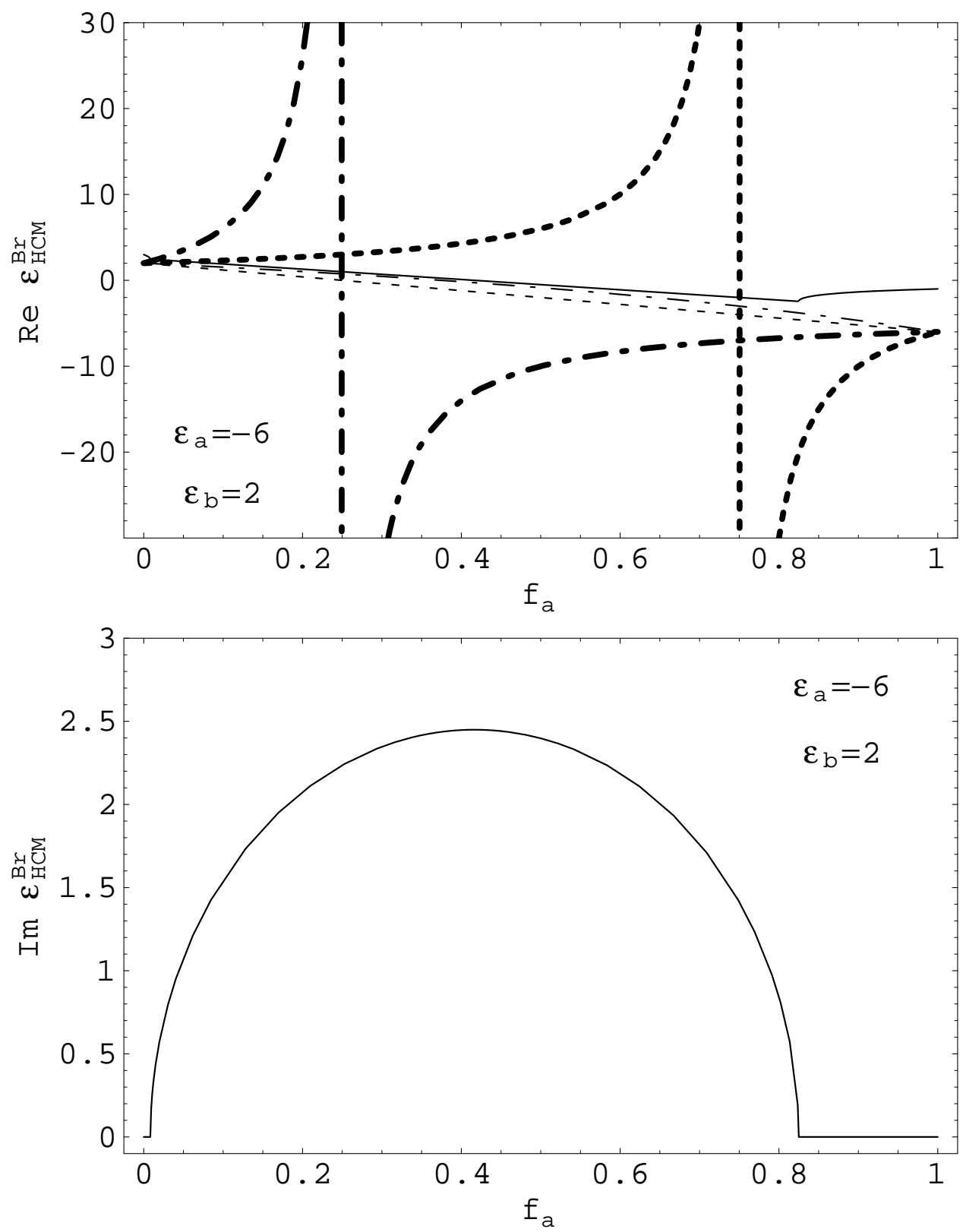

Figure 2: The real (top) and imaginary (bottom) parts of the Bruggeman estimate $\epsilon_{\mathrm{HCM}}^{\mathrm{Br}}$ (solid line) plotted against $f_{a}$ for $\epsilon_{a}=-6$ and $\epsilon_{b}=2$. Also plotted are the real parts of the Wiener bounds, $W_{\alpha}$ (thick dashed line) and $W_{\beta}$ (thin dashed line), and the HashinShtrikman bounds, $H S_{\alpha}$ (thick broken dashed line) and $H S_{\beta}$ (thin broken dashed line). The imaginary parts of $W_{\alpha, \beta}$ and $H S_{\alpha, \beta}$ are null-valued. 

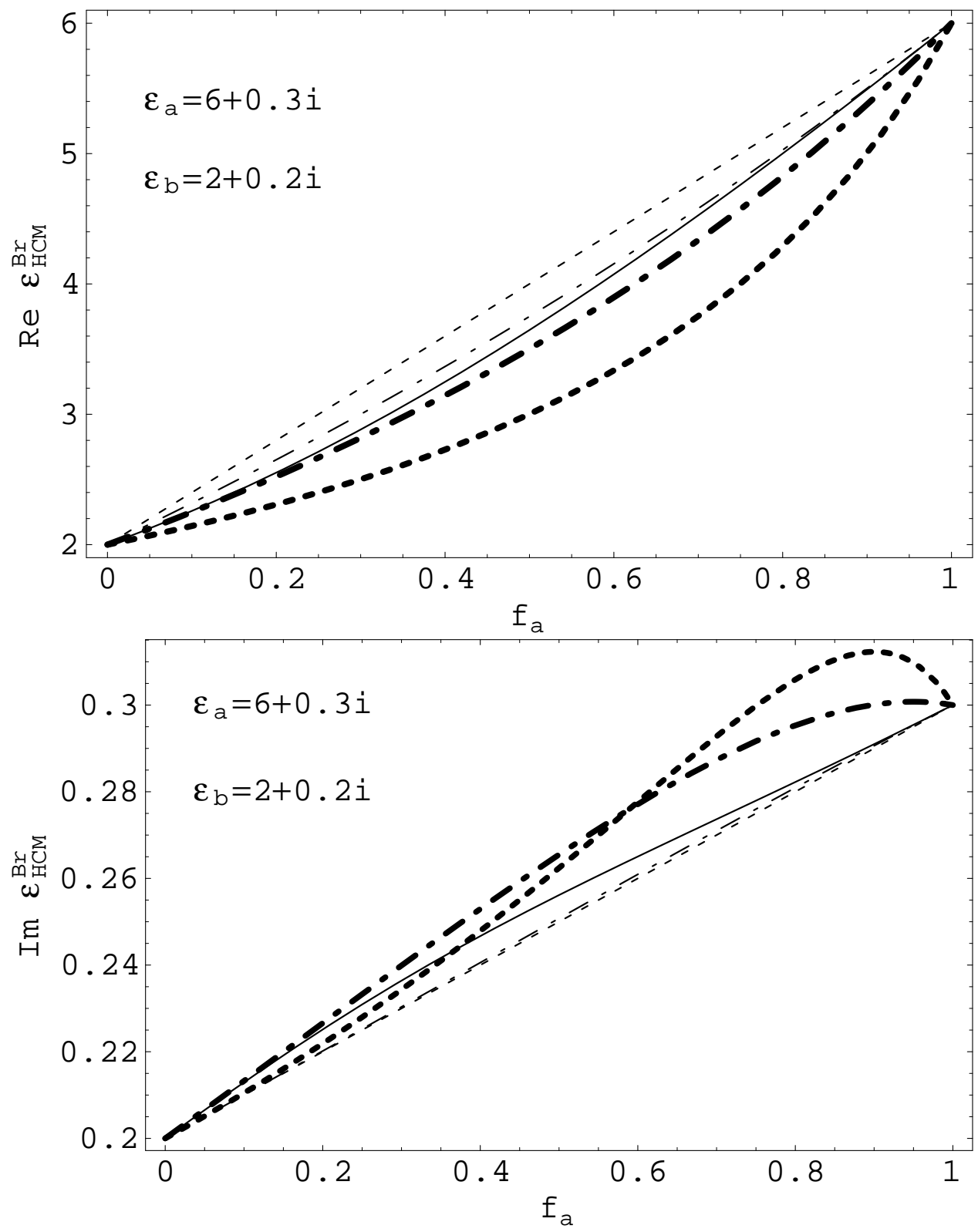

Figure 3: The real (top) and imaginary (bottom) parts of the Bruggeman estimate $\epsilon_{\mathrm{HCM}}^{\mathrm{Br}}$ (solid line) plotted against $f_{a}$ for $\epsilon_{a}=6+0.3 i$ and $\epsilon_{b}=2+0.2 i$. Also plotted are the Wiener bounds, $W_{\alpha}$ (thick dashed line) and $W_{\beta}$ (thin dashed line), and the Hashin-Shtrikman bounds, $H S_{\alpha}$ (thick broken dashed line) and $H S_{\beta}$ (thin broken dashed line). 

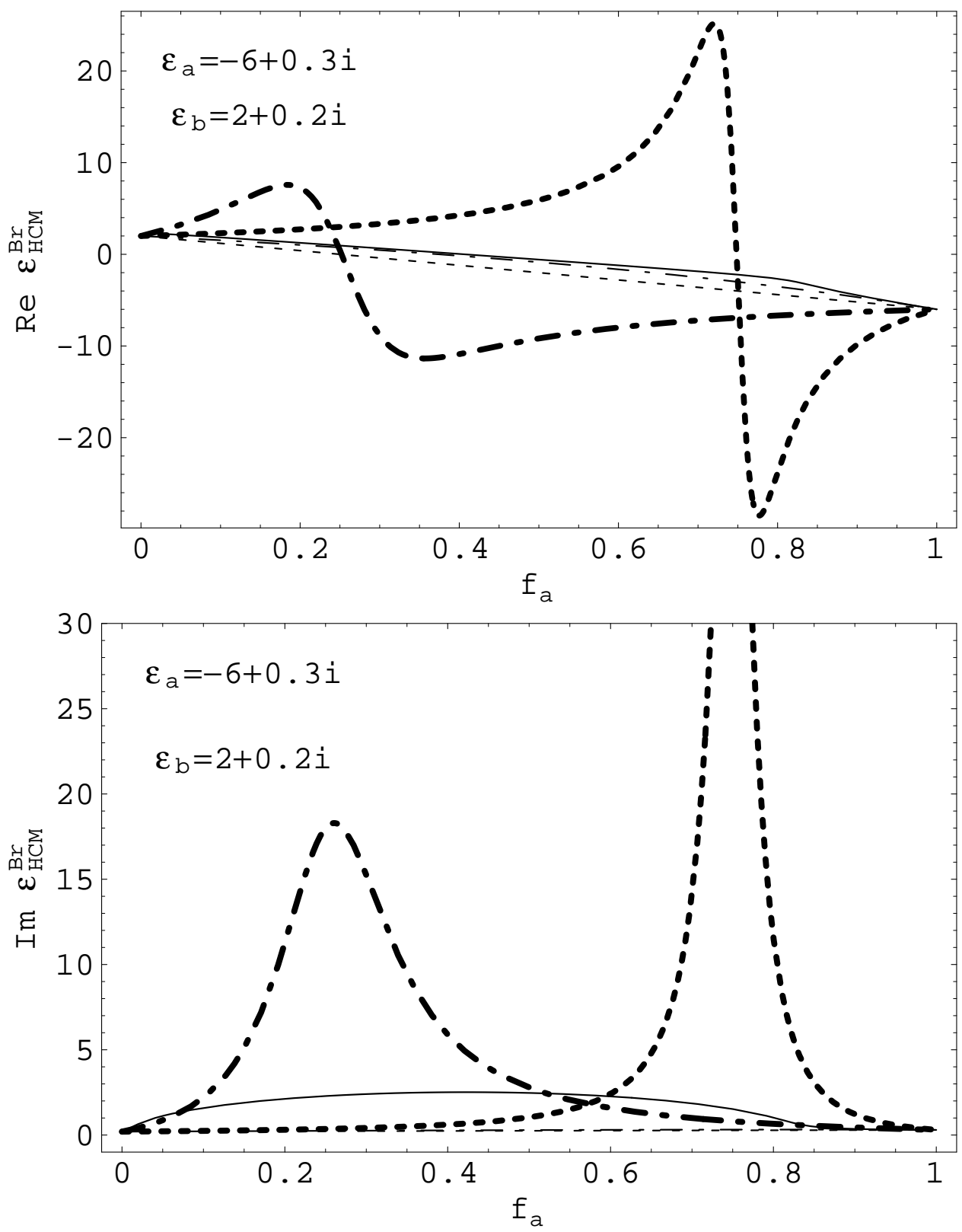

Figure 4: As Figure 3 but for $\epsilon_{a}=-6+0.3 i$ and $\epsilon_{b}=2+0.2 i$. 

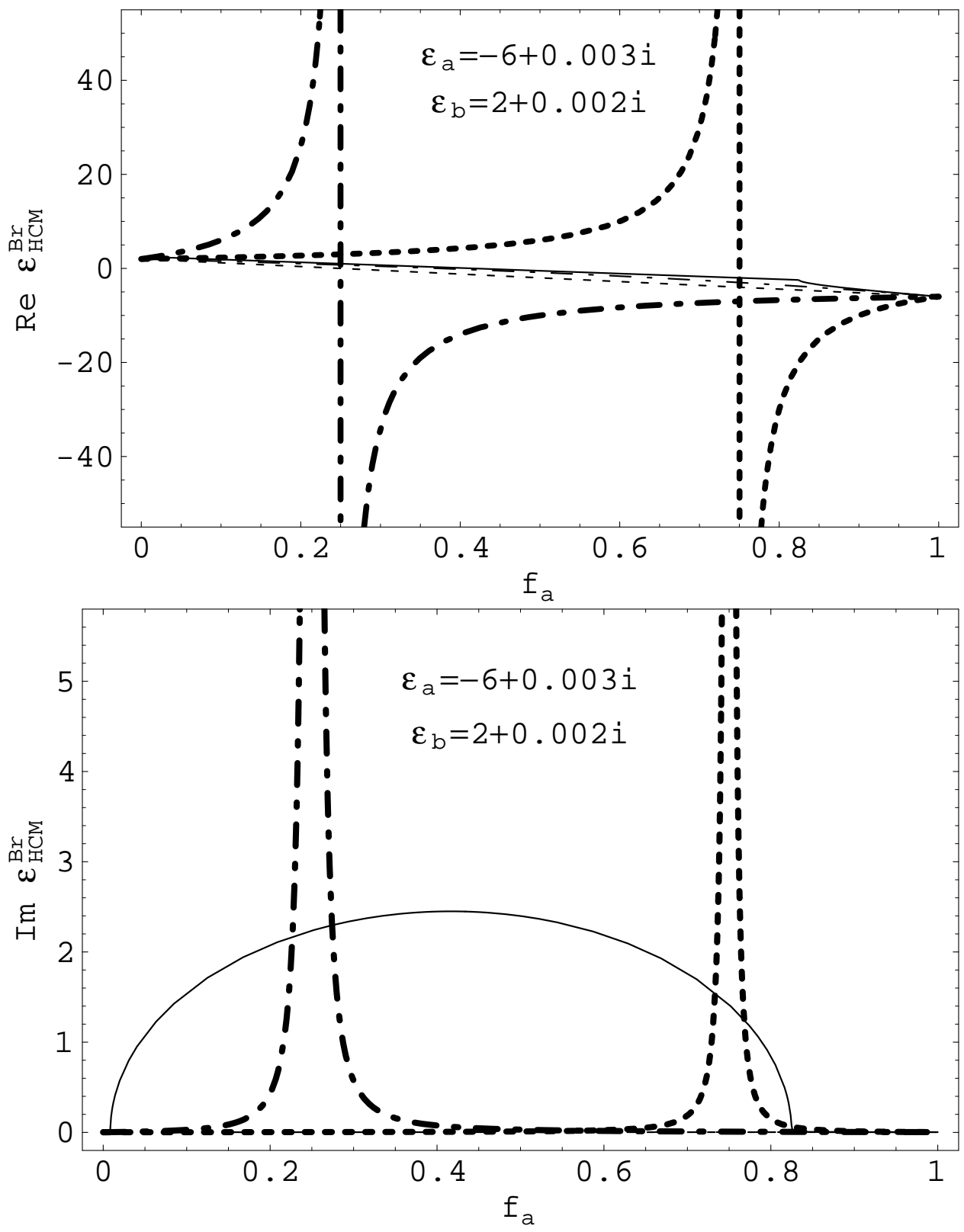

Figure 5: As Figure 3 but for $\epsilon_{a}=-6+0.003 i$ and $\epsilon_{b}=2+0.002 i$. 

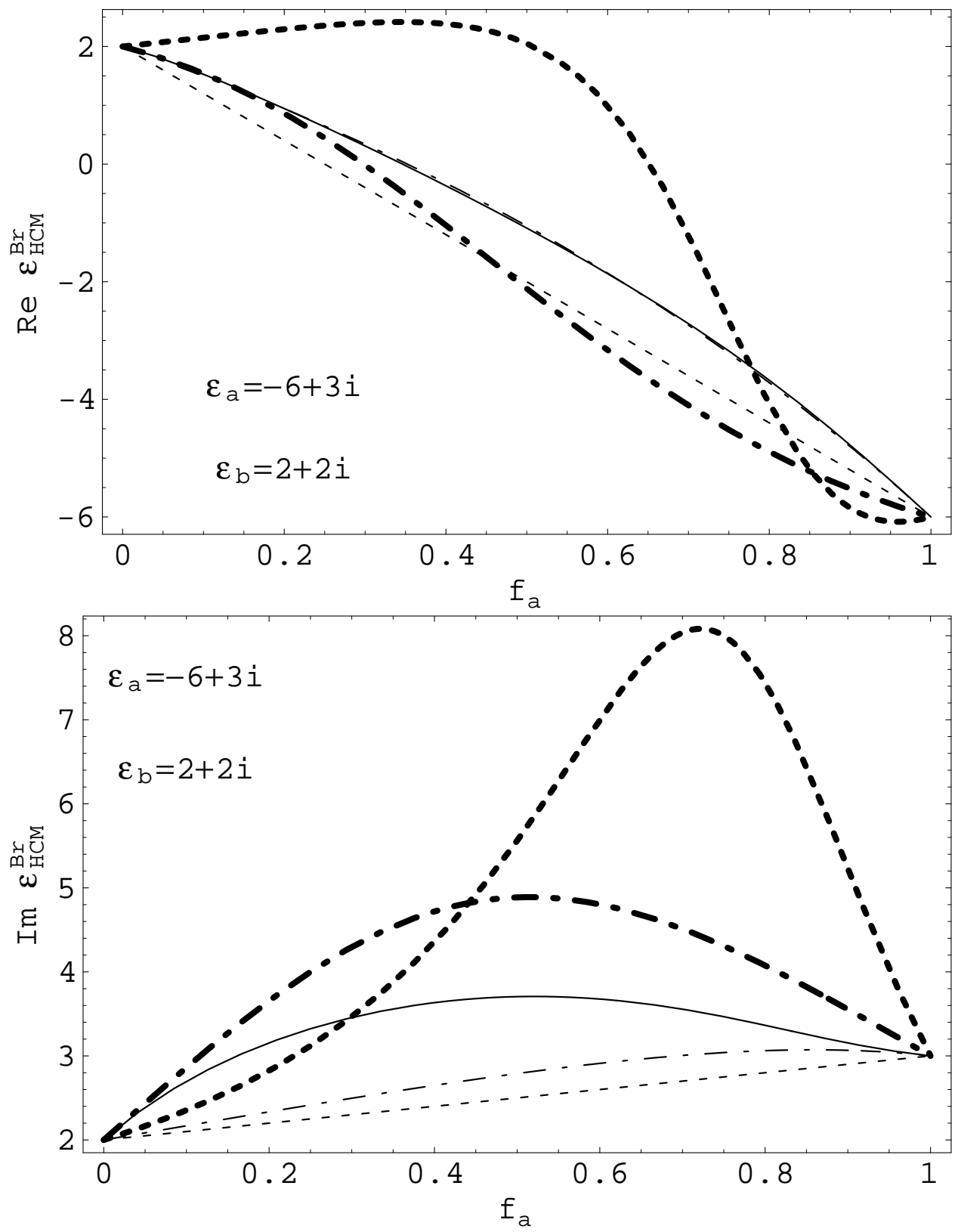

Figure 6: As Figure 3 but for $\epsilon_{a}=-6+3 i$ and $\epsilon_{b}=2+2 i$. 